\title{
A NOTE ON PRINCIPAL SEQUENCES
}

\author{
by B. ROTMAN
}

(Received 21 March, 1963)

A fundamental problem in the theory of ordinals is the assignation of principal sequences to limit numbers of the second number class.

It is our main object here to show that a certain class of methods, which are a natural generalisation of those used in the solution of the corresponding problem for the real numbers (the description of which we omit), must fail to solve the problem. The methods are those which rest on the following assumption: the principal sequence assigned to any limit number of the second number class is determined ance the first $i$ terms of that sequence are known.

Our assertion will follow from Theorem A below.

The following result (see [1], Sect. 9) will be used. If $\phi$ is a function on the second number class such that $\phi(\xi)<\xi$, then there exists an ordinal $\alpha$ for which there are non-denumerably many ordinals $\xi$ such that $\phi(\xi)=\alpha$. (Such functions have been termed regressive functions by Bloch [2], who investigated the precise subsets of the second number class-stationary setswhich are such that any regressive function defined over them produces numbers $\alpha$ with the above property; we shall call these numbers stationary points.)

LEMMA. If $\phi(\xi)<\psi(\xi)<\xi$ for all $\xi<\omega_{1}$, then $\phi$ has at least one stationary point which precedes all the stationary points of $\psi$.

Proof. Consider the smallest stationary point of $\psi$; call this number $\eta^{\psi}$. Let

$$
K\left(\eta^{\psi}\right)=\left\{\xi \mid \psi(\xi)=\eta^{\psi}\right\}
$$

Now $K\left(\eta^{*}\right)$ is non-denumerable, and $\phi\left(K\left(\eta^{\psi}\right)\right)$ is contained in the segment determined by $\eta^{*}$, since $\phi(\xi)<\psi(\xi)$ for all $\xi$. Hence there must exist at least one number $\eta^{\phi}$ which is such that $\left\{\xi \mid \xi \in K\left(\eta^{\psi}\right) \& \phi(\xi)=\eta^{\phi}\right\}$ is non-denumerable, i.e. $\eta^{\phi}$ is a stationary point of $\phi$ which is less than $\eta^{\psi}$. Let us denote by $R\left(\eta^{\phi}\right)$ the set $\left\{\xi \mid \xi \in K\left(\eta^{\psi}\right) \& \phi(\xi)=\eta^{\phi}\right\}$. Then clearly $R\left(\eta^{\phi}\right) \subseteq K\left(\eta^{\psi}\right)$.

THEOREM A. For a given sequence of regressive functions assigning principal sequences to the limit numbers of the second number class, there exists, for any $i<\omega$, a non-denumerable subset of these limit numbers such that the principal sequences assigned to the members of this subset coincide in their first i places.

Proof. Let the principal sequence of $\mu$ be $\left\{f_{i}(\mu)\right\}_{i<\omega}$, where, of course, $f_{1}(\mu)<f_{2}(\mu)<\ldots$ for all $\mu$, and let $\eta_{i}^{i}$ be the $\lambda$ th stationary point of $f_{i}$.

Applying the lemma to $f_{i}$ and $f_{i-1}$, we see that $f_{i-1}$ has a stationary point $\eta_{a_{1}}^{i-1}$ such that $\eta_{a_{1}}^{i-1}<\eta_{1}^{i}$ and $R\left(\eta_{a_{1}}^{i-1}\right) \subseteq K\left(\eta_{1}^{i}\right)$. 
Applying the lemma again to $f_{i-1}$ and $f_{i-2}$, where this time $f_{i-2}\left(R\left(\eta_{a_{1}}^{i-1}\right)\right)$ is considered rather than $f_{l-2}\left(K\left(\eta_{a_{1}}^{i-1}\right)\right)$, we obtain a stationary point $\eta_{a_{2}}^{i-2}$ of $f_{i-2}$ such that

$$
\eta_{a_{2}}^{i-2}<\eta_{a_{1}}^{i-1}<\eta_{1}^{i} \text { and } R\left(\eta_{a_{2}}^{i-2}\right) \subseteq R\left(\eta_{a_{1}}^{i-1}\right) \subseteq K\left(\eta_{1}^{i}\right)
$$

where

$$
R\left(\eta_{a_{2}}^{i-2}\right)=\left\{\xi \mid \xi \in R\left(\eta_{a_{1}}^{i-1}\right) \& f_{1-2}(\xi)=\eta_{a_{2}}^{i-2}\right\}
$$

In all, if we apply the lemma $i-1$ times in the above manner (i.e. using the appropriate $R$ instead of $K$ at each step), we obtain

$$
R\left(\eta_{a_{i-1}}^{1}\right) \subseteq R\left(\eta_{a_{i-2}}^{2}\right) \subseteq \ldots \subseteq R\left(\eta_{a_{1}}^{i-1}\right) \subseteq K\left(\eta_{1}^{i}\right)
$$

where

$$
\eta_{a_{i-1}}^{1}<\eta_{a_{i-2}}^{2}<\ldots<\eta_{a_{1}}^{i-1}<\eta_{1}^{\prime}
$$

$R\left(\eta_{a_{t-1}}^{1}\right)$ is the non-denumerable subset with the stated property; for if $\mu \in R\left(\eta_{a_{1-1}}^{1}\right)$ and $\lambda \in R\left(\eta_{a_{i-1}}^{1}\right)$, then

$$
f_{j}(\mu)=f_{j}(\lambda)=\eta_{a_{-j}}^{j} \text { for } j=1,2, \ldots, i
$$

It is clear that $R\left(\eta_{a_{-1}}^{1}\right)$ is non-denumerable.

The selection of the first stationary point $\eta_{1}^{l}$ in the initial part of the proof was made for simplicity only. The proof will go through if $\eta_{\xi}^{i}$, for any $\xi<\omega_{1}$, is chosen; and hence one could obtain $\boldsymbol{N}_{1}$ distinct sets with the desired property.

Theorem A shows that any method of assigning principal sequences to limit numbers of the second number class which takes the principal sequence as defined, once its first $i$ terms are fixed, must break down when it comes to assign sequences to the members of the nondenumerable set $R\left(\eta_{a_{t-1}}^{1}\right)$. (The case $i=1$ follows from the result quoted above.)

Thus Denjoy's massive attempt [3] to solve the problem of principal sequences must fail, according to his own description of that method. For we find the following assertion in the concluding section of his work (p. 584).

"Let us observe that one of the properties of the system of principal sequences $\dagger$ defined in the preceding pages is that two principal sequences relative to two distinct ordinal numbers cannot have their first two terms in common. The knowledge of the first jump $\left(\alpha_{1}, \alpha_{2}\right)$, in the sequence of ordinal numbers, which is taken by the principal sequence $\left(\alpha_{n}\right)$ of $\alpha$ suffices to determine $\alpha . "$

Returning to Theorem $\mathrm{A}$, we note that it shows that there exists a similarity between principal sequences in the second number class and the " decimal " expansions of real numbers - the property of real numbers corresponding to Theorem A being obvious. Under a suitable restrictive assumption on the functions $f_{i}$, one could state further similarities. For instance, under the assumption that any $\xi$ belongs to $K\left(\eta_{\mu}^{l}\right)$ for some $i<\omega$ and some $\mu<\omega_{1}$, the following theorem holds.

$\dagger$ “Suites canoniques"; Denjoy uses the term "suites principales" for something quite different. 
THEOREM B. For any limit number $\lambda$, the first $i$ terms of the principal sequence of $\lambda$ (for any $i<\omega)$ are shared by non-denumerably many limit numbers.

The proof is immediate.

\section{REFERENCES}

1. H. Bachmann, Transfinite Zahlen (Ergeb. der Math. u. ihrer Grenzgeb., Berlin, 1955).

2. G. Bloch, Sur les ensembles stationnaires de nombres ordinaux et les suites distinguées de fonctions regressives, C. R. Acad. Sc. Paris 236 (1952), 265-268.

3. A. Denjoy, L'Énumération Transfinie, Livre 2: L'arithmétisation du transfini, deuxieme partieLes suites canoniques (Paris, 1952), 439-607.

\section{SiR John CASS COLLEGE}

LONDON 